\title{
Pregnancy-Associated Breast Cancer: Clinicopathological Characteristics of 20 Cases with a Focus on Identifiable Causes of Diagnostic Delay
}

\author{
Derman Basaran $^{\mathrm{a}} \quad$ Mert Turgal $^{\mathrm{b}} \quad$ Kemal Beksac $^{\mathrm{c}}$ Ozgur Ozyuncu ${ }^{\mathrm{b}} \quad$ Omer Aran $^{\mathrm{c}}$ \\ M. Sinan Beksac ${ }^{b}$
}

a Department of Obstetrics and Gynecology, Division of Gynecologic Oncology, Faculty of Medicine, Hacettepe University, Ankara, Turkey

${ }^{\mathrm{b}}$ Department of Obstetrics and Gynecology, Division of Perinatology, Faculty of Medicine, Hacettepe University, Ankara, Turkey

' Department of General Surgery, Faculty of Medicine, Hacettepe University, Ankara, Turkey

Keywords

Pregnancy $\cdot$ Breast cancer · Breast · Diagnosis

\section{Summary}

Background: The primary objective of this study was to evaluate the clinicopathological characteristics of patients with pregnancy-associated breast cancer (PABC), with a special focus on diagnostic delays and the identifiable causes of diagnostic delays. Patients and methods: Clinicopathological data of patients treated for PABC between 2003 and 2012 at Hacettepe University Hospital was retrospectively reviewed. Results: 20 patients with PABC were included. The pathological examination revealed predominance of invasive ductal carcinoma (80\%), grade III tumors (65\%) and advanced-stage (III-IV) disease (75\%). In 8 patients $(40 \%)$, there was a diagnostic delay between occurrence of the presenting symptoms and the initiation of breast mass workup. For these 8 patients, the main identifiable causes of diagnostic delay were the attribution of disease-related symptoms to pregnancy or lactation in 5 (63\%) and negligence of symptoms in 2 (25\%). Conclusions: PABC mostly presents with advanced-stage disease, and there can be a substantial diagnostic delay before these patients receive treatment. Preconceptional, gestational and postpartum examination of women of reproductive age should include a thorough breast examination and should provide adequate information regarding the physiological changes in breast tissue and the possible pathological symptoms.

\section{Introduction}

Breast cancer (BC) is 1 of the most common cancers of both pregnant and non-pregnant women $[1,2]$. The term pregnancy-associated breast cancer (PABC) is used to define a woman who is diagnosed with $\mathrm{BC}$ during her pregnancy, up to 1 year after delivery, or at any time while she is lactating [3]. Although PABC is a relatively rare event with a crude incidence of 1 in 3,000 pregnancies, it is expected that clinicians will encounter these cases more frequently in the near future because of women's increasing propensity to delay childbearing [4]. Previous studies have shown that women with PABC are diagnosed with more advanced-stage disease and larger tumors when compared to non-PABCs [5, 6]. Although this advanced presentation was generally thought to be the result of late diagnosis of patients, in whom the signs and the symptoms of the breast mass is partly obscured by the physiological changes of pregnancy and lactation, there have been very few studies that specifically investigated the possible causes of diagnostic delay in women with PABC [7]. Therefore, our aim in this study was to evaluate the clinicopathological characteristics of patients with $\mathrm{PABC}$ with a special focus on diagnostic delays and the identifiable causes of diagnostic delays.

\section{Patients and Methods}

This retrospective analysis included women diagnosed with $\mathrm{BC}$ either during pregnancy, the first postpartum year, or any time during their lactation period in Hacettepe University Adult Hospital between 2003 and 2012. The following clinical data were recorded from patients' charts and medical records: age, presenting symptom(s), patient-related diagnostic delay (time between presenting symptom(s) and admission to primary

\section{KARGER \\ Fax +49 7614520714 \\ Information@Karger.com}

www.karger.com
(C) 2014 S. Karger GmbH, Freiburg

1661-3791/14/0095-0355\$39.50/0

Accessible online at:

www.karger.com/brc
Derman Basaran, MD

Department of Obstetrics and Gynecology

Hacettepe University Faculty of Medicine

06100, Sihhiye, Ankara, Turkey

dermanbasaran@gmail.com 
Table 1. Clinical and gestational characteristics of 20 patients with $P A B C$

\begin{tabular}{ll}
\hline Parameter & Patients with PABC \\
\hline Patients, n & 20 \\
Age, years (range) & $36.0(28-43)$ \\
Gestational age at diagnosis, weeks (range) $^{\mathrm{a}}$ & $21.5(8-38)$ \\
Time from pregnancy termination or birth to & $7.0(1-24)$ \\
$\quad$ diagnosis, months(range) & \\
Gestational age at delivery, weeks (range) & $36.5(8-39)$ \\
$\begin{array}{l}\text { Pregnancy outcomes, n }(\%) \\
\quad \text { Term delivery }\end{array}$ & $11(55)$ \\
$\quad$ Preterm delivery & $6(30)$ \\
$\quad$ Voluntary termination & \\
\hline
\end{tabular}

${ }^{\mathrm{a}}$ For patients with diagnosis during pregnancy.

${ }^{\mathrm{b}}$ For patients with postpartum diagnosis.

$\mathrm{PABC}=$ pregnancy-associated breast cancer.

${ }^{\mathrm{c}}$ One patient underwent termination before diagnosis of PABC.

physician), physician-related diagnostic delay (time between admission to primary physician and initiation of breast mass workup), any identifiable reasons for diagnostic delay, gestational week at diagnosis, oncological management of BC, management of pregnancy, and maternal and neonatal outcomes. Preterm birth was defined as births occurring before 37 weeks of gestation irrespective of the mode of delivery. Late preterm birth was defined as births occurring between $34^{\circ}$ and $36^{6}$, and early preterm birth was defined as births occurring before $34^{0}$ gestational weeks. Histopathological characteristics of the tumors were recorded from pathology reports. Slides were reviewed if the initial biopsy was performed at another center. Tumor size was obtained from histological reports for patients who were treated with surgery and from initial physical examination records for patients in whom surgery could not be performed. Tumor type, histological grade, estrogen receptor (ER) and progesterone receptor (PR) status, HER2 expression, and nodal status (if available) were also recorded. Disease-free survival (DFS) and overall survival (OS) was defined as the time between pathological diagnosis of $\mathrm{BC}$ and the date of relapse or last known contact, and the date of death caused by disease or last known contact, respectively. SPSS 17.0 (SPSS Inc., Chicago, IL, USA) was used for the data management and statistical analysis. KaplanMeier method was used for the assessment of survival outcomes. As this study represents a retrospective chart review, the Local Ethical Committee permission was not sought. However, all patients signed an informed consent that allows our institution to use their clinical data.

\section{Results}

We identified 20 patients with PABC. Of those, $8(40 \%)$ were diagnosed during pregnancy and $12(60 \%)$ were diagnosed postpartum. The baseline characteristics of the patients are presented in table 1 . The median gestational age of $\mathrm{BC}$ diagnosis in pregnant patients was 21.5 weeks (range 8-38 weeks) and the median time between birth or pregnancy termination and $\mathrm{BC}$ diagnosis was 7.0 months (range 1-24 months) in postpartum diagnosis group. 2 patients with antepartum BC diagnosis opted for voluntary termination of their pregnancy before the second trimester of pregnancy (8th and 13th week). There were 2 early preterm births and 1 late preterm birth in women with antepartum diagnosis. Of these
Table 2. Disease-specific characteristics of 20 patients with $P A B C$

\begin{tabular}{|c|c|}
\hline Variable & Patients with PABC \\
\hline Patients, $\mathrm{n}$ & 20 \\
\hline \multicolumn{2}{|l|}{ Symptoms at diagnosis, $\mathrm{n}(\%)$} \\
\hline Mass & $13(65)$ \\
\hline Mass and pain & $3(15)$ \\
\hline Mass, pain and nipple discharge & $1(5)$ \\
\hline Skin changes on breast & $1(5)$ \\
\hline Low back pain and difficulty in ambulation & $1(5)$ \\
\hline Unknown & $1(5)$ \\
\hline \multicolumn{2}{|l|}{ Identifiable causes of diagnostic delay, n (\%) } \\
\hline No diagnostic delay & $12(60)$ \\
\hline Attributing symptoms to pregnancy & $3(15)$ \\
\hline Attributing symptoms to lactation & $2(10)$ \\
\hline Patients' negligence of symptoms & $2(10)$ \\
\hline False differential diagnosis as mastitis & $1(5)$ \\
\hline \multicolumn{2}{|l|}{ Localization of the breast, $\mathrm{n}(\%)$} \\
\hline Right & $8(40)$ \\
\hline Left & $12(60)$ \\
\hline Tumor size, $\mathrm{cm}$ & $4.9 \pm 3.4$ \\
\hline \multicolumn{2}{|l|}{ Histologic type, n (\%) } \\
\hline Invasive ductal carcinoma & $16(80)$ \\
\hline Mixed (ductal + micropapillary) & $1(5)$ \\
\hline Mixed (ductal + lobular) & $1(5)$ \\
\hline Mucinous carcinoma & $1(5)$ \\
\hline Undifferentiated & $1(5)$ \\
\hline \multicolumn{2}{|l|}{ Histological grade, $\mathrm{n}(\%)$} \\
\hline II & $7(35)$ \\
\hline III & $13(65)$ \\
\hline \multicolumn{2}{|l|}{ Immunohistochemical markers, $\mathrm{n}(\%)$} \\
\hline ER or PR positivity ${ }^{\mathrm{a}}$ & $14(70)$ \\
\hline HER2 expression ${ }^{\mathrm{b}}$ & $5(25)$ \\
\hline Triple negative & $3(15)$ \\
\hline \multicolumn{2}{|l|}{ Nodal status, n (\%) } \\
\hline Positive & $8(40)$ \\
\hline Negative & $7(35)$ \\
\hline Lymph node dissection not performed & $5(25)$ \\
\hline \multicolumn{2}{|l|}{ Clinical stage, $\mathrm{n}(\%)$} \\
\hline II & $5(25)$ \\
\hline III & $8(40)$ \\
\hline IV & $7(35)$ \\
\hline
\end{tabular}

${ }^{a}$ Whenever $10 \%$ or more of tumor cells exhibit ER or PR.

${ }^{\mathrm{b}} \mathrm{A}$ result of $(+++)$ was considered as positive.

$\mathrm{ER}=$ estrogen receptor, $\mathrm{PR}=$ progesterone receptor.

early preterm births, 1 patient delivered dizygotic twins by Csection in the 33rd gestational week after rupture of membranes and the other patient delivered a neonate by emergency C-section because of fetal distress in the 28th gestational week. The latter neonate suffered from respiratory distress syndrome and pneumonia and developed bronchopulmonary dysplasia. There were 3 late preterm births in the postpartum diagnosis group with uneventful neonatal outcomes. There were no other remarkable perinatal complications in the rest of the study group. 
Table 3. Oncological management of 20 patients with PABC

\begin{tabular}{|c|c|c|c|c|c|c|c|c|c|}
\hline Patient & GA Dx & $\begin{array}{l}\text { GAD/ } \\
\text { GAT }\end{array}$ & Stage & Surgery & RT & $\mathrm{CT}$ & DFS (mo) & OS (mo) & Outcome \\
\hline \multicolumn{10}{|c|}{ Antenatal } \\
\hline 1 & $28 w$ & $35 \mathrm{w}$ & II & $\begin{array}{l}\text { Lmp+LND in 30th } \\
\text { week }\end{array}$ & Yes & None & 48 & 48 & Alive with NED \\
\hline 2 & $8 w$ & $8 w$ & IV & $\mathrm{RM}+\mathrm{LND}$ & Yes & PP NEO & 34 & 34 & Alive with NED \\
\hline 3 & $16 \mathrm{w}$ & $28 \mathrm{w}$ & IV & None & Yes & PP NEO & 0 & 11 & Died from metastases \\
\hline 4 & $37 w$ & $38 \mathrm{w}$ & IV & None & Yes & PP ADJ & NA & NA & Lost-to-follow up \\
\hline 5 & $19 \mathrm{w}$ & $37 w$ & IV & None & Yes & $\mathrm{AP}+\mathrm{PP} \mathrm{NEO}$ & 0 & 9 & $\begin{array}{l}\text { Alive with progressive } \\
\text { disease }\end{array}$ \\
\hline 6 & $27 w$ & $33 \mathrm{w}$ & III & $\mathrm{MRM}+\mathrm{LND}$ & Yes & AP NEO & 10 & 10 & Alive with NED \\
\hline 7 & $8 w$ & $13 \mathrm{w}$ & II & Lmp+LND & Yes & PP ADJ & 8 & 8 & Alive with NED \\
\hline 8 & $24 \mathrm{w}$ & $39 w$ & III & $\begin{array}{l}\text { MRM+LND in 24th } \\
\text { week }\end{array}$ & Yes & $\mathrm{AP}+\mathrm{PP} \mathrm{ADJ}$ & 12 & 12 & Alive with disease \\
\hline \multicolumn{10}{|c|}{ Postnatal } \\
\hline 9 & $4 \mathrm{mo}$ & $38 \mathrm{w}$ & II & MRM+LND & None & ADJ & 80 & 80 & Alive with NED \\
\hline 10 & $23 \mathrm{mo}$ & $36 w$ & II & MRM+LND & Yes & ADJ & 65 & 65 & Alive with NED \\
\hline 11 & $23 \mathrm{mo}$ & $39 w$ & III & MRM+LND & Yes & NEO & 20 & 23 & Alive with disease \\
\hline 12 & $4 \mathrm{mo}$ & $8 w$ & III & MRM+LND & Yes & ADJ & 1 & $1 \mathrm{q}$ & Alive with NED \\
\hline 13 & $6 \mathrm{mo}$ & $36 \mathrm{w}$ & III & MRM+LND & Yes & ADJ & 25 & 29 & Died from metastases \\
\hline 14 & $24 \mathrm{mo}$ & $38 \mathrm{w}$ & IV & None & Yes & NEO & 0 & 6 & Died from metastases \\
\hline 15 & $2 \mathrm{mo}$ & $36 \mathrm{w}$ & IV & None & Yes & NEO & 0 & 12 & Died from metastases \\
\hline 16 & $12 \mathrm{mo}$ & $39 w$ & III & $\mathrm{MRM}+\mathrm{LND}$ & Yes & NEO & 51 & 51 & Alive with NED \\
\hline 17 & $8 \mathrm{mo}$ & $37 w$ & II & MRM+LND & Yes & ADJ & 53 & 53 & Alive with NED \\
\hline 18 & $3 \mathrm{mo}$ & $38 \mathrm{w}$ & IV & MRM+LND & Yes & NEO & 20 & 38 & Alive with NED \\
\hline 19 & $1 \mathrm{mo}$ & $39 w$ & III & $\mathrm{RM}+\mathrm{LND}$ & Yes & $\mathrm{AD}$ & 75 & 75 & Alive with NED \\
\hline 20 & $23 \mathrm{mo}$ & $36 w$ & III & $\mathrm{RM}+\mathrm{LND}$ & Yes & NEO & 22 & 22 & Alive with NED \\
\hline
\end{tabular}

GA Dx $=$ Gestational age at diagnosis in AP patients (weeks) or months after delivery in PP patients, $\mathrm{AP}=$ antepartum, $\mathrm{PP}=$ postpartum, GAD/ GAT $=$ gestational age at delivery or termination, $\mathrm{RT}=$ radiotherapy, $\mathrm{CT}=$ chemotherapy, $\mathrm{DFS}=$ disease-free survival, $\mathrm{OS}=$ overall survival, mo $=$ months, $\mathrm{w}=$ weeks, $\mathrm{Lmp}=$ lumpectomy, $\mathrm{LND}=$ lymph node dissection, $\mathrm{NED}=$ no evidence of disease, $\mathrm{ADJ}=$ adjuvant, $\mathrm{NEO}=$ neo-adjuvant, $(\mathrm{M}) \mathrm{RM}=$ (modified) radical mastectomy, NA = not available.

Table 2 shows the disease-specific characteristics of the patients. The major presenting symptoms were self-identified breast mass in $17 / 20(85 \%)$ patients and pain in $4 / 20(20 \%)$ patients. In 8 patients ( $40 \%)$, there was a diagnostic delay between occurrence of the presenting symptoms and the initiation of breast mass workup. All but 1 of these diagnostic lags were patient related. Only 1 patient, who was lactating and had skin changes as presenting symptom, was treated with antibiotics for mastitis, then underwent diagnostic biopsy 1 month after admission to her primary physician. The mean patient-related diagnostic delay was $9.4 \pm 7.5$ months. Attribution of the observed disease-related symptoms to pregnancy or lactation in $5 / 8(63 \%)$ and negligence of symptoms in $2 / 8$ $(25 \%)$ were the main identifiable causes of diagnostic delay. Invasive ductal carcinoma ( $80 \%$ ) was the most common histological type, and majority of the patients $(65 \%)$ had grade III tumors. $70 \%$ of the tumors expressed either ER or PR. HER2 protein overexpression was observed in $25 \%$ of the pathological specimens. Nodal involvement was noted in $53 \%$ of the patients who underwent axillary lymph node dissection. Stage III was the most common stage $(40 \%)$ at presentation and 7 patients $(35 \%)$ had stage IV metastatic disease.
Oncological management of the patients with PABC is summarized in table 3.5 pregnant patients underwent surgery, while the other 3 patients were deemed inoperable due to metastatic disease at presentation. 2 patients underwent lumpectomy with lymph node dissection (LND) and modified radical mastectomy with LND during the 30th and the 24 th week of gestation, respectively. 3 patients underwent surgery after delivery or pregnancy termination. 3 patients with antepartum diagnosis received anthracycline-based chemotherapy (cyclophosphamide, adriamycin and fluorouracil) in second and third trimester of their pregnancies. No significant adverse effects were observed in the pregnant women and their fetuses during and after treatment with chemotherapy. Neonatal examination of these newborns who were subjected to intrauterine chemotherapy was also unremarkable. All but 1 antenatally diagnosed patients were treated with adjuvant radiotherapy after pregnancy. Patients with postpartum diagnosis of $\mathrm{PABC}$ were treated as non-PABC patients with surgery and adjuvant chemoradiation, except in 2 cases with stage IV metastatic BC who received palliative chemotherapy.

Follow-up data was available for 19 patients with a median follow-up time of 22.5 months (range 1-80 months). Median 
DFS and OS was not reached, thus mean DFS and OS were calculated. The mean DFS and OS of the patients with PABC were $60.3 \pm 8.2$ months and $62.4 \pm 7.6$ months, respectively.

\section{Discussion}

In the present retrospective study, we evaluated the clinical and pathological characteristics of 20 patients in whom the diagnosis of the $\mathrm{BC}$ was made during pregnancy or in the first 2 years postpartum in a single tertiary referral center. In our study population, the median age at diagnosis was 36.0 (range 28-43) years, the median gestational age at diagnosis was 21.5 (range 8-38) weeks, and the median time from pregnancy termination or birth to diagnosis was 7.0 (range 1-24) months. These results are consistent with those in previous reports $[3$, $8,9]$. PABC mostly presents as a palpable mass and in rare occasions a bloody nipple discharge may occur [10]. A selfidentified breast mass was the chief complaint in $80 \%$ of our patients followed by pain in $15 \%$. Pregnancy and lactation has significant physiological impacts on breast tissue due to remarkable changes in the hormonal milieu that cause increased breast firmness and nodularity [7, 11]. These physiological changes render clinical examination difficult and, as a consequence, reported delays in diagnosis are common and could range up to 10 months $[7,8,10,12,13]$. According to a comprehensive review by Woo et al. [10], the median diagnostic delay in more recent studies was reported as 1 or 2 months, while older studies described much longer periods of delay. In our study group, the chart review revealed a diagnostic delay in $8 / 20(40 \%)$ of the patients with a mean time from initial symptom to diagnosis of $9.4 \pm 7.5$ months. The analysis of these cases showed that diagnostic delays were mostly due to patient-related factors rather than physician-related clinical underestimation. There was only 1 case of physician-related diagnostic delay, which was resulted from misinterpretation of skin changes of $\mathrm{BC}$ as mastitis of lactation. The other diagnostic delays were due to the patients attributing the disease symptoms to pregnancy (3/8) and lactation (2/8) or the patients not seeking medical care on time (2/8). Our results differ somewhat from those of Taylor et al. [7] who reviewed the diagnostic work-up of 22 women with PABC as a part of large population-based national gestational $\mathrm{BC}$ study. They identified $5(22.7 \%)$ patients with a diagnostic delay of 4-12 weeks and $4(18.2 \%)$ patients with a diagnostic delay of more than 6 months. The authors reported that the cause of diagnostic delay in patients with 4-12 weeks of delay were patient related in 2/5 cases and physician related in 3/5 cases. These findings show that diagnostic delays in PABC could be associated with both physician- and patient-related factors. Preconceptional, gestational and postpartum counselling of women of reproductive age must provide adequate information regarding the physiological changes in breast tissue and the possible disease-related symptoms. It also should be kept in mind that differential diagnosis of a breast lump in pregnancy includes not only cancer but a variety of benign conditions. Thus, a thorough work-up of any mass persisting more than 2 weeks is required before counselling the pregnant woman about the possibility of $\mathrm{BC}[1,10]$. Breast ultrasound is the preferred method of radiological imaging during pregnancy and lactation as it is sensitive, inexpensive and does not present any fetal risks $[1,3,7,10,12]$. If a solid mass is demonstrated by ultrasound, a core biopsy should be performed to exclude malignant disease $[1,3,7,10,12]$.

The pathological examination of our study group revealed predominance of invasive ductal carcinoma (80\%), grade III tumors $(65 \%)$ and advanced stage (III-IV) disease (75\%). These results were similar to those of the previous studies [1, $3,5,6,9,10,14]$. Although PABC was reported to be associated with decreased hormone receptor positivity and increased HER2 expression in previous reports [8, 15, 16], our study group demonstrated a $70 \%$ of ER or PR positivity and a $25 \%$ of HER 2 overexpression, which is comparable with the non-PABC patients [17]. The relatively high percentage of hormone receptor-positive tumors in the present study could be associated with the fact that $60 \%$ of the patients in our study were diagnosed after pregnancy, which might diminish the down-regulating effect of estrogen and progesterone on ER and PR [15]. 55\% of our patients had nodal metastasis, which represents a figure lying at the lower end of the reported incidence of lymphatic involvement in patients with PABC $[9,10]$.

Treatment of $\mathrm{PABC}$ requires a multidisciplinary team including oncosurgeons, medical oncologists, and perinatology specialists with an individualized approach to patients' needs. After a diagnosis of BC, it has been shown that early termination of pregnancy does not improve outcome [1, 9, 10,14]. 2 of our patients underwent voluntary pregnancy termination despite adequate counselling. Surgery is the mainstay of the treatment and has been shown to be safe during all trimesters of pregnancy $[3,18]$. We observed favorable maternal and fetal outcomes in 2 patients who underwent surgery during pregnancy. Adjuvant and neo-adjuvant chemotherapy indications for PABC are similar to that used for non-PABCs $[1,3]$. In our series, 3 patients received anthracycline-based chemotherapy after the first trimester without any materno-fetal complications. Loibl et al. [19] explored the maternal and fetal outcomes of BC treatment in pregnancy in a large cohort and compared the effects of chemotherapy in infants with or without intrauterine exposure. Although infants exposed to intrauterine chemotherapy had statistically non-significant higher rates of perinatal complications compared to infants with no exposure, detailed analysis of these infants revealed that these adverse events were mainly related to premature delivery rather than to chemotherapy exposure. Importantly, cytotoxic agents should only be given to pregnant women after the first trimester since all chemotherapeutic drugs are pregnancy category $\mathrm{D}$ and have teratogenic effects in human 
fetus [10]. Radiotherapy is not a preferred method for treating patients during pregnancy as it is associated with fetal death, malformations, growth restriction, mental retardation and childhood cancers [10]. Therefore, radiation therapy was given to our patients only after termination of pregnancy. Although evaluation of survival outcomes was not a primary objective of our study, and we are aware that a control group is needed to make such a comparison, the mean OS of $60.3 \pm 8.2$ months in our patients with PABC was similar to median OS of 4.9 years (range $0.8-15.9$ years) in 22 patients with $\mathrm{PABC}$ in a case-control study by Ali et al. [20]. They observed that patients with non-PABC have a significantly better OS when compared with PABCs (6.0 vs. 4.9 years, $\mathrm{p}=$ 0.02 ). This finding is supported by a recent meta-analysis including 30 matched case-control studies (3,628 cases and 37,100 controls) [21]. The authors reported that patients with $\mathrm{PABC}$ has a significantly higher risk of death than those with non-PABC (hazard ratio 1.44, 95\% confidence interval $1.27-1.63)$.
In conclusion, our findings confirmed that patients with PABC mostly present with advanced-stage disease and there can be a substantial diagnostic delay before these patients receive treatment. As these diagnostic delays can be associated with both physician- and patient-related factors, education of patients and physicians, particularly obstetricians, about physiological and pathological breast changes during pregnancy is a matter of utmost importance. Preconceptional and antenatal visits are good opportunities for obstetricians to perform a vigilant breast examination and to counsel the patients about the risk of breast cancer.

\section{Disclosure Statement}

There are no conflicts of interest and no relevant sources of funding for this study.

\section{References}

1 Amant F, Deckers S, Van Calsteren K, et al.: Breast cancer in pregnancy: Recommendations of an international consensus meeting. Eur J Cancer 2010;46:3158-3168.

2 Ferlay J, Shin HR, Bray F, et al.: Estimates of worldwide burden of cancer in 2008: GLOBOCAN 2008. Int J Cancer 2010;127:2893-2917.

3 Asgeirsson KS: Pregnancy-associated breast cancer. Acta Obstet Gynecol Scand 2011;90:158-166.

4 Andersson TM, Johansson AL, Hsieh CC, et al.: Increasing incidence of pregnancy-associated breast cancer in Sweden. Obstet Gynecol 2009;114: $568-572$.

5 Anderson BO, Petrek JA, Byrd DR, et al.: Pregnancy influences breast cancer stage at diagnosis in women 30 years of age and younger. Ann Surg Oncol 1996;3:204-211.

6 Beadle BM, Woodward WA, Middleton LP, et al. The impact of pregnancy on breast cancer outcomes in women $\leq 35$ years. Cancer 2009;115:1174 1184

7 Taylor D, Lazberger J, Ives A, et al.: Reducing delay in the diagnosis of pregnancy-associated breast cancer: How imaging can help us. J Med Imaging Radiat Oncol 2011;55:33-42.

8 Bonnier P, Romain S, Dilhuydy JM, et al.: Influence of pregnancy on the outcome of breast cancer: A case-control study. Societe Francaise de Senologie et de Pathologie Mammaire Study Group. Int J Cancer 1997;72:720-727.
9 Halaska MJ, Pentheroudakis G, Strnad P, et al.: Presentation, management and outcome of 32 patients with pregnancy-associated breast cancer: A matched controlled study. Breast J 2009;15:461467

10 Woo JC, Yu T, Hurd TC: Breast cancer in pregnancy: A literature review. Arch Surg 2003;138:9198; discussion 99.

11 Neville MC, McFadden TB, Forsyth I: Hormonal regulation of mammary differentiation and milk secretion. J Mammary Gland Biol Neoplasia 2002; 7:49-66.

12 Doyle S, Messiou C, Rutherford JM, Dineen RA: Cancer presenting during pregnancy: Radiological perspectives. Clin Radiol 2009;64:857-871.

13 Lambe M, Ekbom A: Cancers coinciding with childbearing: Delayed diagnosis during pregnancy? BMJ 1995:311:1607-1608.

14 Cordoba O, Llurba E, Saura C, et al.: Multidisciplinary approach to breast cancer diagnosed during pregnancy: Maternal and neonatal outcomes. Breast 2013;22:515-519.

15 Merkel DE: Pregnancy and breast cancer. Sem Surg Oncol 1996;12:370-375.

16 Elledge RM, Ciocca DR, Langone G, McGuire WL: Estrogen receptor, progesterone receptor, and HER-2/neu protein in breast cancers from pregnant patients. Cancer 1993;71:2499-2506.
17 Viswanathan S, Ramaswamy B: Pregnancy-associated breast cancer. Clin Obstet Gynecol 2011;54: 546-555.

18 Anatolian Medical Oncology Society Group, Ustaalioglu BB, Gumus M, et al.: Malignancies diagnosed during pregnancy and treated with chemotherapy or other modalities (review of 27 cases): Multicenter experiences. Int J Gynecol Cancer 2010;20:698-703.

19 Loibl S, Han SN, von Minckwitz G, et al.: Treatment of breast cancer during pregnancy: An observational study. Lancet Oncol. 2012;13:887-896.

20 Ali SA, Gupta S, Sehgal R, Vogel V: Survival outcomes in pregnancy associated breast cancer: A retrospective case control study. Breast J 2012;18: 139-144.

21 Azim HA Jr, Santoro L, Russell-Edu W, et al.: Prognosis of pregnancy-associated breast cancer: A meta-analysis of 30 studies. Cancer Treat Rev 2012;38:834-842. 